\title{
A Ten-Dimensional Super-Yang-Mills Action with Off-Shell Supersymmetry
}

\author{
Nathan Berkovits \\ Maths Dept., King's College, Strand, London, WC2R 2LS, United Kingdom \\ e-mail: udah101@oak.cc.kcl.ac.uk
}

KCL-TH-93-11

August 1993

\begin{abstract}
After adding seven auxiliary scalar fields, the action for ten-dimensional super-YangMills contains an equal number of bosonic and fermionic non-gauge fields. Besides being manifestly Lorentz and gauge-invariant, this action contains nine spacetime supersymmetries whose algebra closes off-shell. Octonions provide a convenient notation for displaying these symmetries.
\end{abstract}


Ten-dimensional super-Yang-Mills is of special interest to physicists both because it contains the maximum number of supersymmetries consistent with Yang-Mills and because it is described by the massless sector of the ten-dimensional superstring. At the present time, there are two different actions used for describing this theory.

The first action is constructed from a bosonic vector and a fermionic spinor and is both manifestly Lorentz-invariant and gauge-invariant. ${ }^{1}$ However because a vector and a spinor contain different numbers of non-gauge degrees of freedom in ten dimensions, the spacetime supersymmetries of this action only close on-shell. In other words, the commutator of two supersymmetry transformations is not just a spacetime translation plus a gauge transformation, but includes other contributions which only vanish after using equations of motion.

The other action used to describe ten-dimensional super-Yang-Mills is light-cone gauge-fixed and therefore contains only the eight physical bosonic and fermionic fields. ${ }^{2} \mathrm{Al}-$ though eight of the spacetime supersymmetries now close off-shell, this action is non-local in $x^{-}$and neither Lorentz invariance nor gauge invariance is manifest.

In this paper, a new action for ten-dimensional super-Yang-Mills is constructed which combines advantages of these two earlier versions. In addition to being manifestly Lorentz and gauge-invariant, it contains nine spacetime supersymmetries which close off-shell. This action is:

$$
S=\int d^{10} x \operatorname{Tr}\left(-\frac{1}{2} F^{\mu \nu} F_{\mu \nu}+i \psi^{\alpha} \gamma_{\alpha \beta}^{\mu} D_{\mu} \psi^{\beta}-\sum_{j=1}^{7} G_{j} G_{j}\right)
$$

where $D_{\mu}=\partial_{\mu}+g A_{\mu}, F_{\mu \nu}=\left[D_{\mu}, D_{\nu}\right], A_{\mu}$ is the usual vector gauge field, $\psi^{\alpha}$ is the usual sixteen-component Majorana-Weyl spinor, $G_{j}$ is a bosonic auxiliary scalar for $j=1$ to 7 , and all Lie-algebra indices have been suppressed. The above action is invariant under the following supersymmetry transformation:

$$
\begin{gathered}
\delta_{\left[\epsilon^{\alpha}, v_{j}^{\alpha}\right]}^{S} A^{\mu}=i \epsilon \gamma^{\mu} \psi, \quad \delta_{\left[\epsilon^{\alpha}, v_{j}^{\alpha}\right]}^{S} \psi^{\alpha}=-\frac{1}{2}\left(\epsilon \gamma^{\mu \nu}\right)^{\alpha} F_{\mu \nu}+\sum_{j=1}^{7} v_{j}^{\alpha} G_{j}, \\
\delta_{\left[\epsilon^{\alpha}, v_{j}^{\alpha}\right]}^{S} G_{j}=-i v_{j} \gamma^{\mu} D_{\mu} \psi
\end{gathered}
$$


where $\epsilon^{\alpha}$ and $v_{j}^{\alpha}$ are eight bosonic Majorana-Weyl spinors satisfying

$$
v_{j} \gamma^{\mu} v_{k}-\delta_{j k} \epsilon \gamma^{\mu} \epsilon=v_{j} \gamma^{\mu} \epsilon=0
$$

for $j, k=1$ to 7 and $\mu=0$ to 9 (for a given non-zero $\epsilon^{\alpha}$, the $v_{j}^{\alpha}$ 's are determined up to an $\mathrm{SO}(7)$ rotation). Note that $\delta_{\left[\epsilon^{\alpha}, v_{j}^{\alpha}\right]}^{S}$ is fermionic and corresponds to a single supersymmetry generator without the anti-commuting parameter (if desired, $\delta_{\left[\epsilon^{\alpha}, v_{j}^{\alpha}\right]}^{S}$ can be made bosonic by including the anti-commuting parameter $\kappa$, in which case the commutator becomes proportional to $\left.\kappa_{1} \kappa_{2}\right)$.

Using the property that $\frac{1}{2}\left(\epsilon \gamma^{\mu} \epsilon\right) \gamma_{\mu}^{\alpha \beta}=\epsilon^{\alpha} \epsilon^{\beta}+\sum_{j=1}^{7} v_{j}^{\alpha} v_{j}^{\beta}$ and ten-dimensional $\gamma$ matrix identities, it is easy to check that

$$
\begin{gathered}
\delta_{\left[\epsilon^{\alpha}, v_{j}^{\alpha}\right]}^{S} \delta_{\left[\epsilon^{\alpha}, v_{j}^{\alpha}\right]}^{S} A_{\nu}=-i F_{\mu \nu}\left(\epsilon \gamma^{\mu} \epsilon\right), \quad \delta_{\left[\epsilon^{\alpha}, v_{j}^{\alpha}\right]}^{S} \delta_{\left[\epsilon^{\alpha}, v_{j}^{\alpha}\right]}^{S} \psi^{\alpha}=-i D_{\mu} \psi^{\alpha}\left(\epsilon \gamma^{\mu} \epsilon\right), \\
\delta_{\left[\epsilon^{\alpha}, v_{j}^{\alpha}\right]}^{S} \delta_{\left[\epsilon^{\alpha}, v_{j}^{\alpha}\right]}^{S} G_{j}=-i D_{\mu} G_{j}\left(\epsilon \gamma^{\mu} \epsilon\right) .
\end{gathered}
$$

Therefore, for a given $\epsilon^{\alpha}$ and $v_{j}^{\alpha}$, the anti-commutator of $\delta_{\left[\epsilon^{\alpha}, v_{j}^{\alpha}\right]}^{S}$ with itself is proportional to a spacetime translation $\delta_{\epsilon \gamma^{\mu} \epsilon}^{P^{\mu}}$ (i.e., $\left.x^{\mu} \rightarrow x^{\mu}+\epsilon \gamma^{\mu} \epsilon\right)$ plus a gauge transformation $\delta_{A_{\rho}\left(\epsilon \gamma^{\rho} \epsilon\right)}^{\Lambda}$ (i.e., $\left.A_{\mu} \rightarrow A_{\mu}+D_{\mu} A_{\rho}\left(\epsilon \gamma^{\rho} \epsilon\right)\right)$. Furthermore, since the commutator of the Lorentz generator $M_{\mu \nu}$ with $\delta_{\left[\epsilon^{\alpha}, v_{j}^{\alpha}\right]}^{S}$ is equal to $\delta_{\left[\frac{1}{2}\left(\gamma^{\mu \nu} \epsilon\right)^{\alpha}, \frac{1}{2}\left(\gamma^{\mu \nu} v_{j}\right)^{\alpha}\right]}^{S}$,

$$
\begin{gathered}
\left\{\delta_{\left[\epsilon^{\alpha}, v_{j}^{\alpha}\right]}^{S}, \delta_{\left[\frac{1}{2}\left(\gamma^{\mu \nu} \epsilon\right)^{\alpha}, \frac{1}{2}\left(\gamma^{\mu \nu} v_{j}\right)^{\alpha}\right]}^{S}\right\}=\left[M_{\mu \nu}, \delta_{\left[\epsilon^{\alpha}, v_{j}^{\alpha}\right]}^{S} \delta_{\left[\epsilon^{\alpha}, v_{j}^{\alpha}\right]}^{S}\right]=\left[M_{\mu \nu},-i\left(\delta_{\epsilon \gamma^{\rho} \epsilon}^{P^{\rho}}+\delta_{A_{\rho}\left(\epsilon \gamma^{\rho} \epsilon\right)}^{\Lambda}\right)\right] \\
=-i\left(\delta_{\epsilon \gamma^{\rho} \gamma^{\mu \nu} \epsilon}^{P^{\rho}}+\delta_{A_{\rho}\left(\epsilon \gamma^{\rho} \gamma^{\mu \nu} \epsilon\right)}^{\Lambda}\right)
\end{gathered}
$$

So the anti-commutator of $\delta_{\left[\epsilon^{\alpha}, v_{j}^{\alpha}\right]}^{S}$ with $\delta_{\left[\frac{1}{2}\left(\gamma^{\mu \nu} \epsilon\right)^{\alpha}, \frac{1}{2}\left(\gamma^{\mu \nu} v_{j}\right)^{\alpha}\right]}^{S}$ is also proportional to a spacetime translation plus a gauge transformation.

However if $\left[\hat{\epsilon}^{\alpha}, \hat{v}_{j}^{\alpha}\right]$ does not equal $\left[\left(\gamma^{\mu \nu} \epsilon\right)^{\alpha},\left(\gamma^{\mu \nu} v_{j}\right)^{\alpha}\right] f_{\mu \nu}$ for some $f_{\mu \nu},\left\{\delta_{\left[\epsilon^{\alpha}, v_{j}^{\alpha}\right]}^{S}, \delta_{\left[\hat{\epsilon}^{\alpha}, \hat{v}_{j}^{\alpha}\right]}^{S}\right\}$ is not just a translation plus a gauge transformation, but includes other contributions which only vanish on-shell. The maximum number of independent spacetime supersymmetries which closes off-shell is therefore nine, since for more than nine, there always exists at least two supersymmetries, $\delta_{\left[\epsilon^{\alpha}, v_{j}^{\alpha}\right]}^{S}$ and $\delta_{\left[\hat{\epsilon}^{\alpha}, \hat{v}_{j}^{\alpha}\right]}^{S}$, which do not satisfy 
$\left[\hat{\epsilon}^{\alpha}, \hat{v}_{j}^{\alpha}\right]=\left[\left(\gamma^{\mu \nu} \epsilon\right)^{\alpha},\left(\gamma^{\mu \nu} v_{j}\right)^{\alpha}\right] f_{\mu \nu}$ for any $f_{\mu \nu}$. An example of nine independent supersymmetries which do form a closed algebra off-shell is $\delta_{\left[\epsilon^{\alpha}, v_{j}^{\alpha}\right]}^{S}$ and $\delta_{\left[\left(\gamma^{+\mu} \epsilon\right)^{\alpha},\left(\gamma^{+\mu} v_{j}\right)^{\alpha}\right]}^{S}$ for $\mu=1$ to 8 , where $\left(\gamma^{-} \epsilon\right)_{\alpha}=0$ and $\gamma_{\alpha \beta}^{ \pm}=\gamma_{\alpha \beta}^{0} \pm \gamma_{\alpha \beta}^{9}$.

The action of equation (1) is of course analogous to off-shell supersymmetric actions for super-Yang-Mills in three, four, or six dimensions, in which zero, one, or three bosonic auxiliary scalars are added to the bosonic vector and fermionic spinor. Just as the offshell closure of supersymmetry transformations in these dimensions is related to the real, complex, and quaternionic division algebras, ${ }^{3}$ the off-shell closure of the supersymmetry transformations in equation (2) can be related to the octonionic division algebra.

It is well-known that the $\mathrm{SO}(9,1) \gamma$-matrices can be represented as $2 \times 2$ octonionvalued hermitian matrices, $\tilde{\gamma}_{c \dot{d}}^{\mu}$, where $\tilde{\gamma}_{c \dot{d}}^{0}=\sigma_{c \dot{d}}^{0}, \tilde{\gamma}_{c \dot{d}}^{j}=\left(i \sigma_{c \dot{d}}^{2}\right) e_{j}$ for $j=1$ to $7, \tilde{\gamma}_{c \dot{d}}^{8}=\sigma_{c \dot{d}}^{1}$, $\tilde{\gamma}_{c \dot{d}}^{9}=\sigma_{c \dot{d}}^{3}, \sigma_{c \dot{d}}^{r}$ are the usual $2 \times 2$ Pauli-matrices (indices are raised and lowered using the epsilon tensor), and $e_{j}$ for $j=1$ to 7 are the imaginary octonions $\left(e_{j}=-\bar{e}_{j}\right.$ and $e_{j} e_{k}+e_{k} e_{j}=-2 \delta_{j k}$ for $j, k=1$ to 7$)$. In this representation, an $\mathrm{SO}(9,1)$ MajoranaWeyl spinor, $\psi^{\alpha}$, can be written as two octonionic components, $\tilde{\psi}_{1}=\sum_{a=1}^{8} e_{a}\left(\gamma^{+} \psi\right)_{a}$ and $\tilde{\psi}_{2}=\sum_{a=1}^{8} e_{a}\left(\gamma^{-} \psi\right)_{a+8}$, where $e_{8}=1 .^{4}$

If the spacetime supersymmetry parameter, $\epsilon^{\alpha}$, satisfies $\tilde{\epsilon}_{2}=\overline{\tilde{\epsilon}}_{2}$, then the $v_{j}^{\alpha}$ 's can be chosen such that $\tilde{v}_{j}^{c}=e_{j} \tilde{\epsilon}^{c}$ for $c=1$ to 2 and $j=1$ to 7 since

$$
\begin{gathered}
\tilde{v}_{j}^{c}\left(\tilde{\gamma}_{c \dot{d}}^{\mu} \overline{\tilde{v}}_{k}^{\dot{d}}\right)+\left(\tilde{v}_{k}^{c} \tilde{\gamma}_{c \dot{d}}^{\mu}\right) \overline{\tilde{v}}_{j}^{\dot{d}}=\left(e_{j} \tilde{\epsilon}^{c}\right)\left(\tilde{\gamma}_{c \dot{d}}^{\mu}\left(\overline{\tilde{\epsilon}}^{\dot{d}} \bar{e}_{k}\right)\right)+\left(\left(e_{k} \tilde{\epsilon}^{c}\right) \tilde{\gamma}_{c \dot{d}}^{\mu}\right)\left(\overline{\tilde{\epsilon}}^{\dot{d}} \bar{e}_{j}\right)= \\
\tilde{\gamma}_{c \dot{d}}^{\mu}\left(\left(\left(\overline{\tilde{\epsilon}}^{\dot{d}} \bar{e}_{k}\right)\left(e_{j} \tilde{\epsilon}^{c}\right)\right)+\tilde{\gamma}_{c \dot{d}}^{\mu}\left(\left(\left(\overline{\tilde{\epsilon}}^{\dot{d}} \bar{e}_{j}\right)\left(e_{k} \tilde{\epsilon}^{c}\right)\right)=2 \delta_{j k} \tilde{\gamma}_{c \dot{d}}^{\mu}\left(\overline{\tilde{\epsilon}}^{\dot{d}} \tilde{\epsilon}^{c}\right)=2 \delta_{j k} \tilde{\epsilon}^{c}\left(\tilde{\gamma}_{c \dot{d}}^{\mu} \overline{\tilde{\epsilon}}^{\dot{d}}\right),\right.\right.
\end{gathered}
$$

and therefore equation (3) is satisfied. The restriction on $\tilde{\epsilon}_{2}$ comes from the nonassociativity of octonions and reduces the number of independent $\epsilon^{\alpha}$ 's from sixteen to nine.

It is straightforward to show that the spacetime supersymmetry transformations of equation (2) form a closed algebra off-shell when parameterized by these nine $\epsilon^{\alpha}$ 's, and can be expressed in octonionic notation as:

$$
\delta A^{\mu}=\frac{i}{2}\left(\tilde{\epsilon}\left(\tilde{\gamma}^{\mu} \overline{\tilde{\psi}}\right)+\left(\tilde{\psi} \tilde{\gamma}^{\mu}\right) \overline{\tilde{\epsilon}}\right), \quad \delta \tilde{\psi}_{c}=-\frac{1}{2}\left(\left(\tilde{\epsilon} \tilde{\gamma}^{\mu}\right) \tilde{\gamma}^{\nu}\right)_{c} F_{\mu \nu}+\tilde{\epsilon}_{c} G
$$




$$
\delta G=\frac{i}{2}\left(\tilde{\epsilon}\left(\tilde{\gamma}^{\mu} D_{\mu} \overline{\tilde{\psi}}\right)-\left(D_{\mu} \tilde{\psi} \tilde{\gamma}^{\mu}\right) \overline{\tilde{\epsilon}}\right)
$$

where $G=\sum_{j=1}^{7} G_{j} e_{j}$ and contracted $\mathrm{SU}(2)$ spinor indices have been suppressed.

If the octonions in equation (7) are replaced by real, complex, or quaternionic numbers, one obtains the supersymmetry transformations for super-Yang-Mills in three, four, or six dimensions. ${ }^{3}$ Because of associativity of the division algebra, $\tilde{\epsilon}_{2}$ is unrestricted in these dimensions.

There are various possible applications of this new ten-dimensional super-Yang-Mills action. For example, one could try to add more gauge fields so that the anticommutator of two supersymmetry transformations is a pure spacetime translation. This might help in making the off-shell supersymmetry manifest since it would allow non-Wess-Zumino gauge choices. Another possible application would be to construct a new action for tendimensional supergravity such that some of the spacetime super-reparameterizations close off-shell in a similar manner.

It is intriguing that division algebras also play a useful role in understanding the symmetries of the Green-Schwarz superstring, ${ }^{5}$ which describes super-Yang-Mills in firstquantized language. Since string field theory translates first-quantized into secondquantized language, it is natural to conjecture that string field theory for the GreenSchwarz superstring somehow relates the two roles played by the division algebra.

\section{Acknowledgements}

I would like to thank A. Galperin, P. Howe, M. Rocek, J. Schray, W. Siegel, E. Sokatchev, P.Townsend and J. Yamron for useful discussions. This work was supported by an SERC grant.

\section{References}

(1) Brink,L., Schwarz,J.H., and Scherk,J., Nucl.Phys.B121 (1977), p.77.

(2) Brink,L., Lindgren,O., and Nilsson,B.E.W., Nucl.Phys.B212 (1983), p.401. 
(3) Kugo,T. and Townsend,P., Nucl.Phys.B221 (1983), p.357. (1986), p.93.

(4) Chung,K.-W. and Sudbery,A., Phys.Lett.B198 (1987), p.161.

Ramond,P., "Introduction to Exceptional Lie Groups and Algebras", Caltech preprint CALT-68-577 (1976).

(5) Fairlie,D.B. and Manogue,C.A., Phys.Rev.D36 (1987), p.45.

Bengtsson,I. and Cederwall,M., Nucl.Phys.B302 (1988), p.81.

Delduc,F., Galperin,A., Howe,P., and Sokatchev,E., "A twistor formulation of the heterotic $\mathrm{D}=10$ superstring with manifest $(8,0)$ worldsheet supersymmetry", preprint BONN-HE-92-19, JHU-TIPAC-920018, ENSLAPP-L-392-92, July 1992.

Berkovits, N., Phys.Lett.B241 (1990), p.497. 